\title{
An Observational Study in Manipur State, India on Preventive Behavior Influenced by Social Media During the COVID-19 Pandemic Mediated by Cyberchondria and Information Overload
}

\author{
Renu Bala, Amit Srivastava, Gouri Devi Ningthoujam, Thadoi Potsangbam, Amita Oinam, Ch Lily Anal \\ Regional Research Institute for Homoeopathy, Imphal, India
}

Objectives: The coronavirus disease 2019 (COVID-19) pandemic is a public health emergency posing unprecedented challenges for health authorities. Social media may serve as an effective platform to disseminate health-related information. This study aimed to assess the extent of social media use, its impact on preventive behavior, and negative health effects such as cyberchondria and information overload.

Methods: A cross-sectional observational study was conducted between June 10, 2020 and August 9, 2020 among people visiting the outpatient department of the authors' institution, and participants were also recruited during field visits for an awareness drive. Questions were developed on preventive behavior, and the Short Cyberchondria Scale and instruments dealing with information overload and perceived vulnerability were used.

Results: The study recruited 767 participants with a mean age of about 45 years. Most of the participants (>90\%) engaged in preventive behaviors, which were influenced by the extent of information received through social media platforms $(\beta=3.297 ; p<0.001)$ and awareness of infection when a family member tested positive $(\beta=29.082 ; p<0.001)$ or a neighbor tested positive $(\beta=27.964$; $p<0.001)$. The majority (63.0\%) of individuals often searched for COVID-19 related news on social media platforms. The mean \pm standard deviation scores for cyberchondria and information overload were $9.09 \pm 4.05$ and $8.69 \pm 2.56$, respectively. Significant and moderately strong correlations were found between cyberchondria, information overload, and perceived vulnerability to COVID-19.

Conclusions: This study provides evidence that the use of social media as an information- seeking platform altered preventive behavior. However, excessive and misleading information resulted in cyberchondria and information overload.

Key words: COVID-19, Information dissemination, Internet addiction disorder, Preventive health services, Social media

Received: September 21, 2020 Accepted: November 23, 2020

Corresponding author: Amit Srivastava

Regional Research Institute for Homoeopathy, Imphal,

Manipur 795001, India

E-mail: dramit911@gmail.com

This is an Open Access article distributed under the terms of the Creative Commons Attribution Non-Commercial License (https://creativecommons.org/licenses/bync/4.0// which permits unrestricted non-commercial use, distribution, and reproduction in any medium, provided the original work is properly cited.

\section{INTRODUCTION}

A novel coronavirus (now known as severe acute respiratory syndrome coronavirus 2, or SARS-CoV-2) was first reported in Wuhan, Hubei Province, China in December 2019 and has rapidly spread to the rest of the world. The World Health Organization (WHO) declared the disease a pandemic on March 11, 2020 [1]. The coronavirus disease 2019 (COVID-19) pandemic has since reached unprecedented magnitude, with approxi- 
mately more than 29 million cases and around 1 million deaths worldwide, and more than 5 million cases and 83198 deaths in India as of late September 2020 [2]. Governments across the world implemented and enforced quarantine, social distancing, and isolation measures to reduce the risk of infection.

During pandemics, the public needs access to timely and reliable information about the causes, manifestations, and prevention of the disease. Since little is known about COVID-19an issue that was particularly prominent early in the pandemic - it is essential to provide correct information obtained from authentic sources. Mass media have long been considered to be vital shapers of the public's risk perceptions [3]. Currently social media are often seen as fast and effective platforms for searching, sharing, and distributing health information among the general population. Despite the importance of rapid access to information in these critical situations, poor comprehension and inaccurate or false information in the form of rumors or unreliable news can lead to misunderstanding in the community, which makes the situation worse $[4,5]$.

The WHO has recently spoken about the need to fight not only the current COVID-19 pandemic, but also the related infodemic. The perceived quantity and credibility of information received are correlated with adherence to infection prevention behavior [6]. Obsessive online searching for health-related information, typically about specific symptoms, leads to an increase in anxiety or worry that persists as one continues to search; this phenomenon is termed as cyberchondria [7]. Although cyberchondria does not appear to be a distinct disorder, it has been associated with functional impairment and increased healthcare utilization, which has significant public health implications [8]. Information overload is a condition in which one cannot process all the communication and informational input [9].

In the wake of the pandemic, this study aimed to assess the amount of information received online related to COVID-19 from both active searches and passive exposure, preventive behaviors among members of the general public, and the negative health effects of social media usage (namely, cyberchondria and information overload).

\section{METHODS}

\section{Study Setting}

A cross-sectional study was conducted from June 10, 2020 to August 9, 2020 in the city of Imphal in Manipur State, India.
Participants aged 18 years or above who agreed to participate were included in the study.

\section{Study Procedure}

A convenience sampling method involving multiple strategies was used to recruit participants for the study. Patients visiting the general outpatient department of the homeopathic research institute in Imphal, a center of complementary and alternative medicine, were recruited on-site using the assessment questionnaires. People were also approached during an awareness drive for COVID-19 in villages through home-tohome visits and were recruited for the study with their consent.

The socio-demographic characteristics of the subjects were collected in a predesigned format. A self-reported questionnaire on preventive practices was developed following the recommendations of the Ministry of Health and Family Welfare of the government of India [10]. It consisted of 13 questions measured on a 5-point Likert scale with response options of "not at all $=1$," "rarely $=2$," "sometimes $=3$," "very often $=4$," and "always $=5$ ". The numerical responses to each question were added to give a final score that ranged from 13 points to 65 points. Higher scores indicated better preventive practices. The draft questionnaire was sent to 5 experts and the final version was prepared after the consensus of the experts.

There were 18 questions on social media, which were based on several validation studies on cyberchondria, information overload, vulnerability to COVID-19, and the extent of informational support received online [11-14]. The questions were framed to obtain information on the time that participants spent daily on social media platforms, which information they searched for most and how often, and whether they experienced panic due to social media use. Four questions enquired about the extent of information received online about COVID-19 with responses of "didn't receive at all," "received occasionally," "received rarely," "received regularly," and "received a great deal" [11]. Cyberchondria was measured using the Short Cyberchondria Scale, which consisted of 4 questions [12]. Information overload and perceived vulnerability to COVID-19 had 3 questions each $[13,14]$. The response options for all three sections were "completely disagree $=1$," "somewhat disagree $=2$," "somewhat agree $=3$," "mostly agree $=4$," and "completely agree $=5$ ". The numerical responses to the questions in each section were added to give a total score for the respective section, with higher scores indicating a more severe problem.

The questionnaire was in the English language and the par- 
ticipants marked their responses on their own. Any assistance, if needed, was provided by a member of the research team and the response was then cross-checked for completion and correctness.

\section{Statistical Analysis}

The information gathered was entered into a spreadsheet and statistical tests were performed using Microsoft Excel software (Microsoft, Redmond, WA, USA). The descriptive analysis gave frequency, percentage, mean scores, and standard deviation (SD) of responses under each section. Analysis of variance or the $t$-test was performed to identify any significant differences in preventive behavior and social media components depending on demographic variables. The post-hoc test revealed groups that were significantly different from each other. Regression analysis was performed to evaluate the relationships among preventive practices, social media use, and awareness of infection. Pearson correlation analysis was conducted to determine correlations between different social media variables. A $p$-value $<0.05$ was considered to indicate statistical significance.

\section{Ethics Statement}

The study was approved by the Institutional Ethical Committee of the Regional Research Institute for Homoeopathy, Imphal (approval No. 168).

\section{RESULTS}

The study recruited a total of 767 participants, of whom 543 were recruited from the institute's outpatient department and 224 from home-to-home visits. The demographic characteristics of the study participants are given in Supplemental Mate- rial 1. The study participants were evenly distributed among all the age groups, with the highest percentage of participants in the age group of $41-50$ years $(n=178,23.2 \%)$. The mean age of the study participants was 45 years. The majority of participants were women $(n=433,56.4 \%)$, married $(n=622$, $81.1 \%)$, and lived in urban localities $(n=589,76.8 \%)$. Furthermore, $61.7 \%(n=473)$ of the population did not take any prophylactic medicine, while $37.3 \%(n=286)$ of people took homeopathic medicine as a prophylactic measure against COVID-19. Only $13(1.7 \%)$ of the participants had been tested for COVID-19 and only 1 (7.7\%) of those participants had a positive result. At least 1 of the family members of $6(0.8 \%)$ subjects had tested positive, while a neighbor of 17 (2.2\%) participants had tested positive for COVID-19.

\section{Preventive Practices Among the Study Population}

As shown in Supplemental Material 2, that the people of Manipur followed preventive practices to a large extent, such as wearing face masks in public places ( $n=755,98.4 \%)$, avoiding shaking hands and other contacts with people $(n=728$, 93.9\%), and avoiding crowded or public places ( $n=706,92.0 \%)$. The participants were less diligent in cleaning or disinfecting objects and surfaces touched by them or others ( $n=457,59.6 \%$ ). It was observed that preventive practices were significantly associated with the number of hours spent on social media ( $\beta=0.874 ; p=0.003)$, how often subjects sought information on COVID-19 $(\beta=1.387 ; p<0.001)$, the extent of information received online related to COVID-19 $(\beta=3.297 ; p<0.001)$, $c y$ berchondria $(\beta=0.255 ; p<0.001)$, and panic caused by news on COVID-19 in social media $(\beta=3.445 ; p<0.001)$. The most significant relationship with preventive practices was found for an awareness of infection in one's surroundings, as evidenced

Table 1. Regression analysis showing relationships among preventive behavior, social media, and awareness of infection

\begin{tabular}{|c|c|c|c|c|c|}
\hline \multirow{2}{*}{ Preventive behavior } & \multirow{2}{*}{$\beta^{1}$} & \multirow{2}{*}{ SE } & \multicolumn{2}{|c|}{$95 \%$ confidence interval } & \multirow{2}{*}{$p$-value } \\
\hline & & & LCL & UCL & \\
\hline No. of hours spent on social media & 0.874 & 0.292 & 0.301 & 1.447 & 0.003 \\
\hline Frequency of seeking information on COVID-19 on social media & 1.387 & 0.227 & 0.941 & 1.833 & $<0.001$ \\
\hline Extent of information received & 3.297 & 0.268 & 2.770 & 3.824 & $<0.001$ \\
\hline Cyberchondria & 0.255 & 0.059 & 0.140 & 0.370 & $<0.001$ \\
\hline Panic due to social media use & 3.445 & 0.468 & 2.527 & 4.363 & $<0.001$ \\
\hline Family member tested positive for COVID-19 & 29.082 & 0.128 & 28.830 & 29.333 & $<0.001$ \\
\hline Neighbor tested positive for COVID-19 & 27.964 & 0.178 & 27.615 & 28.313 & $<0.001$ \\
\hline
\end{tabular}

SE, standard error of the coefficient; LCL, lower confidence limit; UCL, upper confidence limit; COVID-19, coronavirus disease 2019. ${ }^{1}$ Regression coefficient. 
by a family member testing positive $(\beta=29.082 ; p<0.001)$ or a person in one's neighborhood/locality testing positive ( $\beta=$ 27.964; $p<0.001$ ) (Table 1).

The mean \pm SD score for preventive behavior in the study sample was $58.04 \pm 6.64$ with a $95 \%$ confidence limit of 0.47 .

Table 2. Comparison of mean scores for preventive behavior across demographic variables $(n=767)$

\begin{tabular}{|c|c|c|c|c|}
\hline \multirow{2}{*}{ Variables } & \multirow{2}{*}{ n (\%) } & \multicolumn{3}{|c|}{ Preventive behavior } \\
\hline & & Mean \pm SD & $t / F^{1}$ & $p$-value \\
\hline Total & & $58.04 \pm 6.64$ & & \\
\hline \multicolumn{5}{|l|}{ Age (y) } \\
\hline $18-30$ & $155(20.2)$ & $58.94 \pm 6.38$ & 1.935 & 0.103 \\
\hline $31-40$ & $147(19.2)$ & $57.72 \pm 7.60$ & & \\
\hline $41-50$ & $178(23.2)$ & $58.57 \pm 5.64$ & & \\
\hline $51-60$ & $166(21.6)$ & $57.12 \pm 6.85$ & & \\
\hline$>60$ & $121(15.8)$ & $57.78 \pm 6.66$ & & \\
\hline \multicolumn{5}{|l|}{ Gender } \\
\hline Men & $334(43.5)$ & $57.33 \pm 6.76$ & -2.601 & 0.009 \\
\hline Women & $433(56.4)$ & $58.59 \pm 6.49$ & & \\
\hline \multicolumn{5}{|l|}{ Marital status } \\
\hline Married & $622(81.1)$ & $57.83 \pm 6.67$ & -1.890 & 0.060 \\
\hline Unmarried & $145(18.9)$ & $58.96 \pm 6.43$ & & \\
\hline \multicolumn{5}{|l|}{ Type of area } \\
\hline Rural & $135(17.6)$ & $59.43 \pm 6.63$ & 5.787 & 0.003 \\
\hline Urban & $589(76.8)$ & $57.60 \pm 6.66$ & & \\
\hline Semi-urban & $43(5.6)$ & $59.77 \pm 5.40$ & & \\
\hline \multicolumn{5}{|l|}{ Education } \\
\hline Up to junior high school & $116(15.1)$ & $57.35 \pm 7.50$ & 2.525 & 0.040 \\
\hline High school & $156(20.3)$ & $58.19 \pm 6.93$ & & \\
\hline Higher secondary & $131(17.1)$ & $56.71 \pm 6.80$ & & \\
\hline Graduate & $304(39.6)$ & $58.72 \pm 6.04$ & & \\
\hline Postgraduate and above & $60(7.8)$ & $58.48 \pm 6.23$ & & \\
\hline \multicolumn{5}{|l|}{ Occupation } \\
\hline Government job & 130 (16.9) & $58.64 \pm 6.45$ & 3.590 & 0.007 \\
\hline Business & $129(16.8)$ & $58.04 \pm 6.32$ & & \\
\hline Private job & $151(19.7)$ & $56.30 \pm 6.77$ & & \\
\hline Homemaker & $225(29.3)$ & $58.36 \pm 6.72$ & & \\
\hline Unemployed & $132(17.2)$ & $58.91 \pm 6.56$ & & \\
\hline \multicolumn{5}{|l|}{ Type of family } \\
\hline Nuclear & $269(35.1)$ & $59.44 \pm 5.70$ & 4.596 & $<0.001$ \\
\hline Joint & $498(64.9)$ & $57.29 \pm 6.98$ & & \\
\hline \multicolumn{5}{|l|}{ Chronic illness } \\
\hline Yes & $174(22.7)$ & $57.94 \pm 6.20$ & 1.355 & 0.259 \\
\hline No & $548(71.4)$ & $57.94 \pm 6.79$ & & \\
\hline Don't know & $45(5.9)$ & $59.62 \pm 6.31$ & & \\
\hline
\end{tabular}

SD, standard deviation.

${ }^{1} t$-test or analysis of variance.
Preventive practices were significantly more commonly practiced by women $(p=0.009)$ and persons living in a nuclear family $(p<0.001)$. Significant differences in practices were found among demographic groups according to the type of area $(p=0.003)$, education $(p=0.040)$, and occupation $(p=0.007)$ (Table 2). The post-hoc analysis showed that subjects living in rural areas (vs. urban areas, $Q=4.113 ; p=0.010$ ), with a graduate education (vs. higher secondary, $Q=4.109 ; p=0.031$ ), with a government job (vs. a private job, $Q=4.185 ; p=0.026$ ), as well as homemakers/housewives (vs. private job, $Q=4.192$; $p=0.026$ ) and those who were unemployed (vs. private job, $\mathrm{Q}=4.689 ; p=0.008$ ), showed significantly higher levels of preventive practices (Table 3 ).

\section{Social Media Use}

The majority of the participants $(n=395,51.5 \%)$ spent on an average upto 1 hour a day on social media platforms and $63.0 \%$ $(n=483)$ of individuals searched for COVID-19-related news on these platforms during the pandemic. Fewer than half (43.3\%) of the participants believed that COVID-19 news on social media spread panic among the public. To a greater extent, information on scientific facts related to the pandemic was received by $58.5 \%(n=449)$, on preventing contracting the virus by $49.7 \%$ $(n=381)$, on the spread of the virus by $49.3 \%(n=378)$, and resources to give and receive social support during the pandemic by $41.7 \%(n=320)$ of participants. With the array of information available on social media platforms, the majority of people

Table 3. Post-hoc analysis of preventive behavior, cyberchondria, information overload, and perceived vulnerability

\begin{tabular}{lcc}
\hline Preventive behavior & Q-value $^{\mathbf{1}}$ & p-value \\
\hline Rural vs. urban & 4.113 & 0.010 \\
Higher secondary vs. graduate & 4.109 & 0.031 \\
Government job vs. private job & 4.185 & 0.026 \\
Private job vs. homemaker & 4.192 & 0.026 \\
Private job vs. unemployed & 4.689 & 0.008 \\
Cyberchondria & & \\
Rural vs. semi-urban & 4.550 & 0.004 \\
Urban vs. semi-urban & 4.090 & 0.011 \\
Information overload & & \\
$\quad$ Junior high vs. higher secondary & 4.255 & 0.023 \\
Chronic illness: yes vs. not sure & 3.611 & 0.029 \\
Perceived vulnerability & & \\
Rural vs. urban & 3.505 & 0.036 \\
Urban vs. semi-urban & 3.519 & 0.035 \\
\hline
\end{tabular}

${ }^{1}$ Tukey honest significant difference q-statistics. 
experienced cyberchondria, as $42.8 \%(n=328)$ felt frightened, $42.1 \%(n=323)$ felt frustrated, 34.2\% $(n=262)$ felt confused, and $35.7 \%(n=274)$ felt that once they started reading information about COVID-19 online it was hard for them to stop. There was information overload about COVID-19, as shown by the fact that $48.2 \%(n=370)$ of the participants were distracted by the excessive amount of information, $72.5 \%(n=556)$ were overwhelmed by the amount of information that they processed daily, and $82.5 \%(n=633)$ participants could not form a coherent picture of what was happening. A small number of partici-

Table 4. Comparison of mean scores of cyberchondria, information overload, perceived vulnerability across demographic variables $(n=767)$

\begin{tabular}{|c|c|c|c|c|c|c|c|c|c|}
\hline \multirow{2}{*}{ Variables } & \multicolumn{3}{|c|}{ Cyberchondria } & \multicolumn{3}{|c|}{ Information overload } & \multicolumn{3}{|c|}{ Perceived vulnerability } \\
\hline & Mean \pm SD & $t / F^{1}$ & $p$-value & Mean \pm SD & $t / \mathbf{F}^{1}$ & $p$-value & Mean \pm SD & $t / F^{1}$ & $p$-value \\
\hline Total & $9.09 \pm 4.05$ & & & $8.69 \pm 2.56$ & & & $6.65 \pm 2.40$ & & \\
\hline Age (y) & & 0.587 & 0.672 & & 1.284 & 0.275 & & 1.210 & 0.305 \\
\hline $18-30$ & $9.29 \pm 4.61$ & & & $8.37 \pm 3.02$ & & & $7.08 \pm 2.82$ & & \\
\hline $31-40$ & $8.83 \pm 4.08$ & & & $8.58 \pm 2.57$ & & & $7.29 \pm 2.352$ & & \\
\hline $41-50$ & $9.29 \pm 3.90$ & & & $8.97 \pm 2.36$ & & & $7.63 \pm 2.36$ & & \\
\hline $51-60$ & $9.18 \pm 3.67$ & & & $8.79 \pm 2.29$ & & & $7.45 \pm 2.08$ & & \\
\hline$>60$ & $8.75 \pm 4.00$ & & & $8.68 \pm 2.55$ & & & $7.48 \pm 2.32$ & & \\
\hline Gender & & -1.792 & 0.074 & & 1.261 & 0.208 & & 2.487 & 0.013 \\
\hline Men & $8.79 \pm 4.36$ & & & $8.82 \pm 2.73$ & & & $7.64 \pm 2.66$ & & \\
\hline Women & $9.33 \pm 3.79$ & & & $8.58 \pm 2.42$ & & & $7.20 \pm 2.16$ & & \\
\hline Marital status & & 0.897 & 0.371 & & 2.562 & 0.011 & & 2.607 & 0.010 \\
\hline Married & $9.16 \pm 3.95$ & & & $8.81 \pm 2.46$ & & & $7.51 \pm 2.32$ & & \\
\hline Unmarried & $8.80 \pm 4.47$ & & & $8.14 \pm 2.92$ & & & $6.88 \pm 2.66$ & & \\
\hline Type of area & & 5.235 & 0.006 & & 2.439 & 0.088 & & 5.543 & 0.004 \\
\hline Rural & $8.64 \pm 3.26$ & & & $8.29 \pm 2.15$ & & & $6.98 \pm 1.84$ & & \\
\hline Urban & $9.07 \pm 4.24$ & & & $8.80 \pm 2.70$ & & & $7.54 \pm 2.52$ & & \\
\hline Semi-urban & $10.91 \pm 3.15$ & & & $8.42 \pm 1.50$ & & & $6.60 \pm 1.88$ & & \\
\hline Education & & 0.886 & 0.472 & & 2.742 & 0.028 & & 1.363 & 0.245 \\
\hline Up to junior high school & $8.71 \pm 3.78$ & & & $8.18 \pm 2.38$ & & & $7.02 \pm 1.89$ & & \\
\hline High school & $9.03 \pm 3.35$ & & & $8.58 \pm 2.25$ & & & $7.51 \pm 2.20$ & & \\
\hline Higher secondary & $9.50 \pm 3.91$ & & & $9.16 \pm 2.51$ & & & $7.62 \pm 2.31$ & & \\
\hline Graduate & $9.00 \pm 4.40$ & & & $8.65 \pm 2.79$ & & & $7.43 \pm 2.689$ & & \\
\hline Postgraduate and above & $9.60 \pm 4.69$ & & & $9.10 \pm 2.43$ & & & $7.08 \pm 2.34$ & & \\
\hline Occupation & & 0.587 & 0.672 & & 1.657 & 0.158 & & 0.956 & 0.431 \\
\hline Government job & $8.85 \pm 4.30$ & & & $8.58 \pm 2.73$ & & & $7.40 \pm 2.60$ & & \\
\hline Business & $8.82 \pm 4.03$ & & & $8.79 \pm 2.55$ & & & $7.72 \pm 2.55$ & & \\
\hline Private job & $9.09 \pm 4.07$ & & & $9.06 \pm 2.58$ & & & $7.40 \pm 2.30$ & & \\
\hline Homemaker & $9.40 \pm 3.53$ & & & $8.67 \pm 2.18$ & & & $7.32 \pm 1.98$ & & \\
\hline Unemployed & $9.08 \pm 4.61$ & & & $8.30 \pm 2.94$ & & & $7.17 \pm 2.77$ & & \\
\hline Type of family & & 2.430 & 0.015 & & -1.492 & 0.136 & & 0.485 & 0.628 \\
\hline Nuclear & $9.59 \pm 4.24$ & & & $8.51 \pm 2.30$ & & & $7.45 \pm 2.31$ & & \\
\hline Joint & $8.83 \pm 3.92$ & & & $8.78 \pm 2.70$ & & & $7.36 \pm 2.44$ & & \\
\hline Chronic illness & & 0.893 & 0.410 & & 3.834 & 0.022 & & 2.033 & 0.132 \\
\hline Yes & $9.13 \pm 4.13$ & & & $9.07 \pm 2.49$ & & & $7.71 \pm 2.37$ & & \\
\hline No & $9.15 \pm 4.06$ & & & $8.63 \pm 2.62$ & & & $7.31 \pm 2.44$ & & \\
\hline Don't know & $8.31 \pm 3.60$ & & & $7.98 \pm 1.95$ & & & $7.18 \pm 1.81$ & & \\
\hline
\end{tabular}

SD, standard deviation.

${ }^{1} t$-test or analysis of variance. 
Table 5. Correlations between cyberchondria, information overload, and perceived vulnerability

\begin{tabular}{lcc}
\hline Variables & $\boldsymbol{r}^{\mathbf{1}}$ & $\boldsymbol{p}$-value \\
\hline Cyberchondria and information overload & 0.595 & $<0.001$ \\
Cyberchondria and perceived vulnerability & 0.503 & $<0.001$ \\
Information overload and perceived vulnerability & 0.588 & $<0.001$
\end{tabular}

'Pearson correlation coefficient.

pants ( $n=217,28.2 \%)$ agreed that they were vulnerable to contracting COVID-19 in the given circumstances (Supplemental Material 3). The mean $\pm S D$ scores of cyberchondria, information overload, and perceived vulnerability were $9.09 \pm 4.05,8.69 \pm$ 2.56 , and $6.65 \pm 2.40$, respectively. Cyberchondria was significantly higher among nuclear families $(p=0.015)$ and information overload was experienced more by married individuals $(p=0.011)$. Men $(p=0.013)$ and married persons $(p=0.010)$ perceived significantly higher vulnerability to COVID-19. Significant differences were found in scores for cyberchondria ( $p=0.006)$ and perceived vulnerability $(p=0.004)$ by type of area. Information overload was significantly different among various education groups $(p=0.028)$ and in persons with chronic illnesses $(p=0.022$ ) (Table 4). The post-hoc analysis revealed that participants living in semi-urban areas (vs. rural, $Q=4.550$; $p=0.004$; and vs. urban, $\mathrm{Q}=4.090 ; p=0.011$ ) showed significantly higher cyberchondria, while those living in urban areas (vs. rural, $\mathrm{Q}=3.505 ; p=0.036$; and vs. semi-urban, $\mathrm{Q}=3.519$; $p=0.035$ ) experienced significantly higher vulnerability to $\mathrm{CO}$ VID-19. Similarly, information overload was significantly higher among persons with education up to higher secondary (vs. junior high school, $Q=4.255 ; p=0.023$ ) and those with chronic illnesses (vs. not sure, $\mathrm{Q}=3.611 ; p=0.029$ ) (Table 3). Moderately strong and positive correlations were found between cyberchondria and information overload $(r=0.595 ; p<0.001)$; cyberchondria and perceived vulnerability $(r=0.503 ; p<0.001)$; and between information overload and perceived vulnerability $(r=0.588, p<0.001)$ (Table 5).

\section{DISCUSSION}

In recent years, a series of infectious disease outbreaks around the world have shed light on the significance of effective communication strategies [15]. An emerging infectious disease threatens the health of large numbers of people and requires urgent action to stop the disease at the community level [16]. Difficulties in an outbreak's initial stage mainly arise from high levels of uncertainty about the exact route of contamination, treatment, and recovery [17]. In this study, the amount of COVID-19 related information participants received online, from both active searches and passive exposure of social media, was associated with the adoption of preventive behaviors. On average, participants were middle-aged (around 45 years) and the predominant gender was woman. In previous studies, it has been stated that women access social networks more frequently than men [18].

During recent infectious disease outbreaks, social media networking sites have functioned as first-hand information channels from which the public can obtain disease-related information [19]. Although the role of social media has been established, the question of how informational support received might lead to an overload of information for an individual, which in turn may result in cyberchondria, remains unanswered. This study showed a correlation between information overload and cyberchondria, which aligns with the findings of another study [11]. Furthermore, studies investigating the public's response to the information received and searched during such outbreaks are limited. To fill this gap, this study examined how social media use influenced the degree to which members of the public received correct information, which in turn predicted their preventive behaviors and resulted in cyberchondria. The extent of information received online in this study led to fear, frustration, and confusion, which were aggregately termed as "cyberchondria," among $42.8 \%, 42.1 \%$, and $34.2 \%$ of participants, respectively. Self-relevant emotions such as fear or anger can strongly shape people's beliefs about how risks influence them and mediate the influence of media exposure on personal-level risk perception and, in turn, increase desirable preventive behaviors $[20,21]$. This fact was corroborated by the present study, which showed a significant relationship between cyberchondria and preventive behavior $(p<0.001)$.

In this study, the majority of participants (63.0\%) only read COVID-19 related information on social media platforms, spending up to 1 hour (51.5\%), 2 hours (25.3\%), and 3 hours or more (23.2\%) per day on these platforms. Recent research has also showed that people rely heavily on the Internet to search for relevant COVID-19 health information [22,23]. Besides acquiring information through active searching, people are also incidentally exposed to health information online [24]. The remarkable overall preventive behavior among the participants of this study is consistent with findings from other studies on individuals' engagement in preventive measures against COVID-19 
$[25,26]$. Similar to other studies, the practices followed by more than $90 \%$ participants were covering one's mouth while sneezing/coughing, wearing a facemask when in public places [27,28], avoiding shaking hands with other people, avoiding crowded places [28], avoiding non-essential social and cultural events, and avoiding public transport and non-essential travels [27]. Although the majority of participants showed good preventive practices, disinfecting surfaces or objects was found to be lacking $(59.6 \%)$, perhaps because disinfection requires particular effort. Overall, some preventive measures require greater effort (e.g., cleaning frequently touched surfaces) or pose greater difficulties (e.g., staying home) than others and are thus deemed less feasible.

It has been documented that fearful people tend to perceive greater risk because they have a sense of uncertainty and little control over their situations [29,30]. The health belief model, protection motivation theory, and precaution adaption process model have theorized that a person's perceived risk of a particular health hazard motivates him or her to engage in preventive behaviors as a way to reduce the risk [31-33]. According to the inverted U-shaped fear drive model, a moderate level of fear can engender a motivational state for adaptive coping behaviors, but when fear levels are too low or high; individuals may not attend to or avoid such behaviors [34]. This finding was substantiated in the present study, as the majority of people experienced a moderate level of fear (mean, 2.37 \pm 1.12), which led to preventive behavior in more than $90 \%$ of people.

Awareness of infection in one's social surroundings, including family, friends and relatives, and local communities, are likely to affect people's risk perceptions and their engagement in preventive behaviors [11]. The findings of this study showed a significant and strong relationship between preventive practices and awareness of infection, such as a family member testing positive $(\beta=29.082 ; p<0.001)$ and a person in the neighborhood/locality testing positive $(\beta=27.964 ; p<0.001)$.

Using social media as an information source increases both cyberchondria and information overload and the extent of information received online had an impact on fear among members of the public, which was found to motivate the adoption of preventive practices. Furthermore, people who experience cyberchondria and information overload feel more vulnerable and at risk of contracting COVID-19. This study attempted to extend the connections among social media use, risk perception, and preventive behaviors. However, some limitations of this work should be considered. As this was a cross-sectional study, we could not assess the causal relationship between preventive practices and social media exposure. This study was conducted mostly among people visiting a healthcare facility, which may limit the generalizability of the results to the general population. Nonetheless, participants were also recruited from home-to-home visits during an awareness drive for COVID-19, which balanced the study sample. Another limitation of this study is that the Likert scale used in the questionnaire may have had a bias towards agreement, as there was no midpoint (neutral).

Social media platforms have emerged to be a necessary tool for the public, as they help information to quickly reach people at all levels. Through social media, the health system and resources of India have provided access to knowledge and information about COVID-19 among a larger population. However, the perceptions of this information by the population need to be evaluated, as negative effects are emerging. The purpose of this study was to examine the effects of social media on preventive behavior adopted by the general population of Imphal during the COVID-19 pandemic, as mediated by the negative effects of social media (namely, cyberchondria and information overload). It follows from the findings that, although cyberchondria and information overload are generally regarded to be negative, during the COVID-19 pandemic they contributed to the widespread adoption of recommended preventive behavior by the population of Imphal. The finding that using social media as a source of information gave rise to both cyberchondria and information overload invites further research on the impact of social media on human behavior, especially during pandemics.

\section{SUPPLEMENTAL MATERIALS}

Supplemental materials are available at https://doi.org/10. 3961/jpmph.20.465.

\section{CONFLICT OF INTEREST}

The authors have no conflicts of interest associated with the material presented in this paper.

\section{FUNDING}

None. 


\section{ACKNOWLEDGEMENTS}

None.

\section{AUTHOR CONTRIBUTIONS}

Conceptualization: RB, AS. Data curation: AS. Formal analysis: AS. Funding acquisition: None. Methodology: RB, AS. Project administration: RB, TP, GDN, CLA, AO. Visualization: AS. Writing - original draft: RB, AS, TP, GDN, CLA, AO. Writing - review \& editing: AS, RB.

\section{ORCID}

Renu Bala https://orcid.org/0000-0002-5965-7491

Amit Srivastava https://orcid.org/0000-0002-3485-5424

Gouri Devi Ningthoujam https://orcid.org/0000-0001-56484399

Thadoi Potsangbam https://orcid.org/0000-0003-2915-7995

Amita Oinam https://orcid.org/0000-0002-0164-8947

Ch Lily Anal https://orcid.org/0000-0002-7244-9742

\section{REFERENCES}

1. World Heath Organization. Coronavirus disease (COVID-19) pandemic [cited 2020 Sep 18]. Available from: https://www. euro.who.int/en/health-topics/health-emergencies/coronavirus-covid-19/novel-coronavirus-2019-ncov.

2. World Health Organization. WHO coronavirus disease (COVID19) dashboard [cited 2020 Sep 18]. Available from: https://covid19.who.int/.

3. Snyder LB, Rouse RA. The media can have more than an impersonal impact: the case of AIDS risk perceptions and behavior. Health Commun 1995;7(2):125-145.

4. Sahni H, Sharma H. Role of social media during the COVID-19 pandemic: beneficial, destructive, or reconstructive? Int J Acad Med 2020;6(2):70-75.

5. Hickok K. Husband and wife poison themselves trying to selfmedicate with chloroquine. Live Science; 2020 Mar 24 [cited 2020 Sep 18]. Available from: https://www.livescience.com/ coronavirus-chloroquine-self-medication-kills-man.html.

6. Lep Ž, Babnik K, Hacin Beyazoglu K. Emotional responses and self-protective behavior within days of the COVID-19 outbreak: the promoting role of information credibility. Front Psychol 2020;11:1846.
7. Menon V, Kar SK, Tripathi A, Nebhinani N, Varadharajan N. Cyberchondria: conceptual relation with health anxiety, assessment, management and prevention. Asian J Psychiatr 2020; 53:102225.

8. Mathes BM, Norr AM, Allan NP, Albanese BJ, Schmidt NB. Cyberchondria: overlap with health anxiety and unique relations with impairment, quality of life, and service utilization. Psychiatry Res 2018;261:204-211.

9. Beaudoin CE. Explaining the relationship between internet use and interpersonal trust: taking into account motivation and information overload. J Comput Mediat Commun 2008; 13(3):550-568.

10. Government of Karnataka. Detail question and answers on COVID-19 for public [cited 2020 Sep 17]. Available from: https:// www.mohfw.gov.in/pdf/FAQ.pdf.

11. Li S, Feng B, Liao W, Pan W. Internet use, risk awareness, and demographic characteristics associated with engagement in preventive behaviors and testing: cross-sectional survey on COVID-19 in the United States. J Med Internet Res 2020;22(6): e19782.

12. Jokić-Begić N, Mikac U, Čuržik D, Jokić CS. The development and validation of the short cyberchondria scale (SCS). J Psychopathol Behav Assess 2019;41(4):662-676.

13. Karr-Wisniewski P, Lu Y. When more is too much: operationalizing technology overload and exploring its impact on knowledge worker productivity. Comput Human Behav 2010;26(5): 1061-1072.

14. Farooq A, Laato S, Islam AN. Impact of online information on self-isolation intention during the COVID-19 pandemic: crosssectional study. J Med Internet Res 2020;22(5):e19128.

15. Parmer J, Baur C, Eroglu D, Lubell K, Prue C, Reynolds B, et al. Crisis and emergency risk messaging in mass media news stories: is the public getting the information they need to protect their health? Health Commun 2016:31(10):1215-1222.

16. Würz A, Nurm ÜK, Ekdahl K. Enhancing the role of health communication in the prevention of infectious diseases. J Health Commun 2013;18(12):1566-1571.

17. Lin L, McCloud RF, Bigman CA, Viswanath K. Tuning in and catching on? Examining the relationship between pandemic communication and awareness and knowledge of MERS in the USA. J Public Health 2017;39(2):282-289.

18. Psylla I, Sapiezynski P, Mones E, Lehmann S. The role of gender in social network organization. PLoS One 2017;12(12):e0189873.

19. Jang K, Baek YM. When information from public health officials is untrustworthy: the use of online news, interpersonal net- 
works, and social media during the MERS outbreak in South Korea. Health Commun 2019;34(9):991-998.

20. Dunlop S, Wakefield M, Kashima Y. Can you feel it? Negative emotion, risk, and narrative in health communication. Media Psychol 2008;11(1):52-75.

21. Paek HJ, Oh SH, Hove T. How fear-arousing news messages affect risk perceptions and intention to talk about risk. Health Commun 2016;31(9):1051-1062.

22. Ahmad AR, Murad HR. The impact of social media on panic during the COVID-19 pandemic in Iraqi Kurdistan: online questionnaire study. J Med Internet Res 2020;22(5):e19556.

23. Cuan-Baltazar JY, Muñoz-Perez MJ, Robledo-Vega C, PérezZepeda MF, Soto-Vega E. Misinformation of COVID-19 on the internet: infodemiology study. JMIR Public Health Surveill 2020;6(2):e18444.

24. Tian Y, Robinson JD. Incidental health information use on the internet. Health Commun 2009;24(1):41-49.

25. Wise T, Zbozinek TD, Michelini G, Hagan CC, Mobbs D. Changes in risk perception and self-reported protective behaviour during the first week of the COVID-19 pandemic in the United States. R Soc Open Sci 2020;7(9):200742.

26. Zhong BL, Luo W, Li HM, Zhang QQ, Liu XG, Li WT, et al. Knowledge, attitudes, and practices towards COVID-19 among Chinese residents during the rapid rise period of the COVID-19 outbreak: a quick online cross-sectional survey. Int J Biol Sci
2020;16(10):1745-1752.

27. Kwok KO, Li KK, Chan HH, Yi YY, Tang A, Wei WI, et al. Community responses during early phase of COVID-19 epidemic, Hong Kong. Emerg Infect Dis 2020;26(7):1575-1579.

28. Jungmann SM, Witthöft M. Health anxiety, cyberchondria, and coping in the current COVID-19 pandemic: which factors are related to coronavirus anxiety? J Anxiety Disord 2020;73:102239.

29. Lerner JS, Keltner D. Beyond valence: toward a model of emotion-specific influences on judgement and choice. Cogn Emot 2000;14(4):473-493.

30. Lerner JS, Gonzalez RM, Small DA, Fischhoff B. Effects of fear and anger on perceived risks of terrorism: a national field experiment. Psychol Sci 2003;14(2):144-150.

31. Rosenstock IM. Historical origins of the health belief model. Health Educ Monogr 1974;2(4):328-335.

32. Rogers RW. A protection motivation theory of fear appeals and attitude change1. J Psychol 1975;91(1):93-114.

33. Weinstein ND. Unrealistic optimism about susceptibility to health problems: conclusions from a community-wide sample. J Behav Med 1987;10(5):481-500.

34. Janis IL. Effects of fear arousal on attitude change: recent developments in theory and experimental research. In: Berkowitz L, editor. Advances in experimental social psychology: Vol. 3. New York: Academic Press; 1967, p. 166-224. 